\title{
Associated Factors and Comorbidities of Airflow Limitation in Subjects Undergoing Comprehensive Health Examination in Japan - Survey of Chronic Obstructive Pulmonary Disease Patients Epidemiology in Japan (SCOPE- J)
}

This article was published in the following Dove Press journal: International Journal of Chronic Obstructive Pulmonary Disease

\author{
Hisamitsu Omori (D) \\ Noritaka Higashi ${ }^{2}$ \\ Takeshi Nawa ${ }^{3}$ \\ Toshiki Fukui ${ }^{4}$ \\ Toshihiko Kaise (D) $^{5}$ \\ Takeo Suzuki $\left.{ }^{5}\right)^{5}$ \\ 'Department of Biomedical Laboratory \\ Sciences, Faculty of Life Sciences, \\ Kumamoto University, Kumamoto, Japan; \\ ${ }^{2}$ Department of Respiratory Medicine, \\ Japanese Red Cross Kumamoto Health \\ Care Center, Kumamoto, Japan; ${ }^{3} \mathrm{Nawa}$ \\ Clinic, Hitachi, Japan; ${ }^{4}$ Center for \\ Preventive Medical Treatment, Olive \\ Takamatsu Medical Clinic, Takamatsu, \\ Japan; ${ }^{5}$ Japan Development Division, \\ GlaxoSmithKline K.K., Tokyo, Japan
}

Purpose: To identify associated factors of having at least one of the airflow limitation, chronic cough/phlegm, and currently treated respiratory diseases in health examinees, and to describe the characteristics of each subgroup classified by comorbidities.

Subjects and Methods: This was an observational cross-sectional survey carried out in multiple regions of Japan. Subjects aged 40 years older, undergoing comprehensive health examination, were recruited. Airflow limitation was defined as having forced expiratory volume in $1 \mathrm{~s}$ /forced vital capacity lower than $70 \%$. Associated factors of having at least one of the airflow limitation, chronic cough/phlegm, and currently treated respiratory diseases were examined by logistic regression analysis. Subgroup classification by comorbidity patterns was conducted by hierarchical cluster analysis.

Results: In a total of 22,293 subjects, $1520(6.8 \%)$ had at least one of the airflow limitation, chronic cough/phlegm, and currently treated respiratory diseases. With this objective variable, the following explanatory variables were significantly associated: older age, higher total score in the chronic obstructive pulmonary disease assessment test (CAT) and coexistence of lung cancer (common in ever-smokers and never-smokers), higher pack-years, lower body mass index, higher C-reactive protein, without coexistence of diabetes mellitus (specific in ever-smokers), male sex, coexistence of anxiety, and sleep disorder (specific in neversmokers). Among the 1520 subjects, 1512 subjects with smoking history data were classified by comorbidity patterns into subgroups of "no comorbidities," "mixed comorbidities," "inflammatory comorbidities," "overweight," "underweight," and "chronic kidney disease." "Inflammatory comorbidities" were specific in ever-smokers, and "underweight" was specific in never-smokers.

Conclusion: Several factors were identified as associated factors of having at least one of airflow limitation, chronic cough/phlegm, and currently treated respiratory diseases and they were different between ever-smokers and never-smokers. Different comorbidity patterns were observed by smoking history. These findings could provide information to assist the management of subjects with COPD or at risk for COPD in the general population.

Keywords: spirometry, lung function, general population, health checkup, cluster analysis, clinical characteristics
Correspondence: Toshihiko Kaise Japan Development Division,

GlaxoSmithKline K., I-8-I Akasaka,

Minato-ku, Tokyo 107-0052, Japan

Tel +8I 8059279500

Fax +81342315993

Email toshihiko.kaise@gsk.com
International Journal of Chronic Obstructive Pulmonary Disease 2020:15 3039-3050

3039

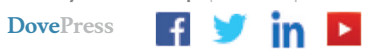

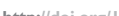




\section{Introduction}

This is the follow-up report of the study SCOPE-J (Survey of Chronic Obstructive pulmonary disease Patients Epidemiology in Japan). In the previous reports, we demonstrated the prevalence and characteristics of airflow limitation and chronic cough/phlegm among subjects undergoing comprehensive health examination. ${ }^{1,2}$ Comprehensive health examination in Japan includes spirometry testing and provides an opportunity for collecting spirometry data along with other clinical characteristics within the general population. Typically, subjects undergoing comprehensive health examination are company employees and non-employee community residents.

In this study, subjects with COPD or at risk for COPD in the general population were identified by spirometry data and self-administered questionnaire responses in comprehensive health examination settings. In addition to subjects with airflow limitation, those with chronic cough/ phlegm or under treatment for respiratory diseases were considered at risk for COPD. The objectives of this study were examining associated factors for such conditions and identifying clusters of comorbidities among such subjects, by aiming for providing information to assist the management of subjects with COPD or at risk for COPD in the general population.

The major risk factor for chronic obstructive pulmonary disease (COPD) is smoking history, but COPD also occurs among never-smokers. The proportion of neversmokers among COPD patients has been reported to be approximately $25-45 \%$ in developed and developing countries. $^{3}$ In our survey, SCOPE-J, 31\% of subjects with airflow limitation were never-smokers. ${ }^{1}$ Since associated factors of airflow limitation among never-smokers could be different from those among ever-smokers, ${ }^{3,4}$ in the present study, we analyzed factors associated with having at least one of airflow limitation, chronic cough/ phlegm, and respiratory diseases stratified by smoking history.

A wide range of comorbidities have been associated with COPD, including cardiovascular disease, hypertension, metabolic syndrome, diabetes, osteoporosis, musculoskeletal diseases, lung cancer, anxiety, and depression. ${ }^{5-8}$ Additionally, the impacts of comorbid conditions on the clinical and economic burdens of COPD have been reported.${ }^{9-12}$ Systemic inflammation, as well as aging and smoking, contribute to the development of many COPD-related comorbidities. ${ }^{13}$ Recent studies classified COPD patients into subgroups, based on the clustering of comorbidities, and examined the characteristics of the subgroups. ${ }^{14,15}$ Yet, this approach has been conducted only among COPD patients and has not been reported among subjects with COPD or at risk for COPD in the general population.

\section{Subjects and Methods Study Design and Participants}

The study SCOPE-J was a prospective, multicenter, crosssectional, observational study. Fourteen sites participated from the following regions in Japan: five in Kanto, one in Chubu, two in Kinki, three in Chugoku/Shikoku, and three in Kyushu. Subjects were recruited between December 2012 and August 2013. The following parameters were investigated in health examinees aged 40 years and older: age; sex; height; weight; smoking history; spirometry findings; blood pressure; white blood cell count; levels of high sensitivity C-reactive protein (CRP); lipids, fasting blood glucose, hemoglobin A1c (HbA1c), and creatinine; symptoms of cough/phlegm, history and current treatment status for respiratory diseases (chronic bronchitis, pulmonary emphysema, COPD, bronchial asthma, diffuse panbronchiolitis, bronchiectasis, pneumonia, pulmonary tuberculosis, pulmonary fibrosis, lung abscess, and pneumoconiosis); comorbidities (myocardial infarction, angina pectoris, cerebrovascular diseases, osteoporosis, fracture, depression, anxiety disorder, sleep disorder, diabetes mellitus, and lung cancer); and CAT findings. ${ }^{16}$

The CAT was originally developed with the aim of developing a better understanding among both COPD patients and physicians in daily clinical practice on how the condition of the disease was affecting the health and daily living of the patients. Additionally for research purposes, the CAT has also been used in evaluating the health status and respiratory symptoms of a wider population including the general population ${ }^{17}$ and healthy working population. ${ }^{18}$ In our study, we asked all the participants regardless of having COPD to fill out the CAT questionnaire to evaluate respiratory symptoms and health status.

This study was conducted in accordance with the Declaration of Helsinki and the Ethical Guidelines for Epidemiological Research (partially revised on December 1, 2008 by the Ministry of Education, Culture, Sports, Science and Technology and the Ministry of Health, Labour and Welfare in Japan), with the approval of the Ethics Committee of the Faculty of Life Sciences, Kumamoto University (Number 575). The health 
examinees that participated in the study provided written informed consent.

\section{Procedures}

Spirometer quality control and calibration followed previously published recommendations. ${ }^{19}$ All participating sites in this study were members of the Japan Society of Ningen Dock (ie the Japanese language of comprehensive health examination) and routinely performed standardized, high-quality spirometry. The airway reversibility test by bronchodilator inhalation was not carried out, because the objective of health examination was screening and not diagnosis.

Airflow limitation was defined as FEV1/FVC ([forced expiratory volume in $1 \mathrm{~s}] /[$ forced vital capacity]) lower than $70 \%$. The predictive value of lung function was calculated using the following predictive equation (male: FEV1 $[\mathrm{L}]=0.036 \times$ height $[\mathrm{cm}]-0.028 \times$ age -1.178 FVC $[\mathrm{L}]=0.042 \times$ height $[\mathrm{cm}]-0.024 \times$ age -1.785 ; female: FEV $1[\mathrm{~L}]=0.022 \times$ height $[\mathrm{cm}]-0.022 \times$ age $0.005 ; \mathrm{FVC}[\mathrm{L}]=0.031 \times$ height $[\mathrm{cm}]-0.019 \times$ age 1.105 ) reported by the Japanese Respiratory Society. ${ }^{20}$

Smoking history, chronic symptoms of cough/phlegm, diagnosis history, and current treatment of respiratory diseases and comorbidities were evaluated by a self-administered questionnaire.

Both past and current smokers were included in the ever-smokers group when classified by the smoking status. Regardless of the pack-years, when subjects answered that they were smoking or had smoked regularly, we regarded them as "ever-smokers."

Chronic cough/phlegm was defined as cough and phlegm lasting more than 3 months in a year and for more than 2 years. $^{21}$

Criteria for comorbidities were as follows according to those reported by the Japan Society of Ningen Dock: overweight, body mass index (BMI) $\geq 25 \mathrm{~kg} / \mathrm{m}^{2}$; underweight BMI $<18.5 \mathrm{~kg} / \mathrm{m}^{2}$; inflammation, CRP $>0.3 \mathrm{mg} / \mathrm{dL}$ or white blood cell count $>8500 / \mu \mathrm{L}$; chronic kidney disease (CKD), estimated glomerular filtration rate (eGFR) $<60 \mathrm{~mL} / \mathrm{min} / 1.73 \mathrm{~m}^{2}{ }^{22}$ The eGFR was calculated using the estimation equation reported by the Japanese Society of Nephrology: eGFR $\left(\mathrm{mL} / \mathrm{min} / 1.73 \mathrm{~m}^{2}\right)=194 \times$ serum creatinine $(\mathrm{Cr})^{-1.094} \times \mathrm{age}^{-0.287}$ for male and $194 \times$ $\mathrm{Cr}^{-1.094} \times$ age $^{-0.287} \times 0.739$ for female. ${ }^{23}$ A comorbidity was defined to be present if the subject had any treatment for the particular comorbidity at the time of health examination. Information on current treatment status for respiratory diseases and comorbidities was collected from subjects using questionnaires. Lung cancer comorbidity was considered to be present if a subject had a history of lung cancer, regardless of undergoing treatment at the time of health examination.

\section{Statistical Analysis}

We used logistic regression analysis (fixed adjusted model) to examine associations between having at least one of the airflow limitation, chronic cough/phlegm, and currently treated respiratory diseases (described in the section "Study design and participants") and potential associated factors. Potential associated factors included age, sex, pack-years, BMI, CRP, CAT score, myocardial infarction, angina pectoris, cerebrovascular disease, osteoporosis, fracture, depression, anxiety disorder, sleep disorder, diabetes, and lung cancer. In calculating adjusted odds ratios of age, sex, pack-years, BMI, CRP, and CAT score, we used fixed adjusted models with these six explanatory variables ( 5 variables for never-smokers, after excluding pack-years). Adjusted odds ratios for comorbidities were additionally calculated by applying age, sex, pack-years, and BMI as explanatory variables (excluding pack-years for never-smokers).

In the analysis of association with diabetes, post hoc analyses were performed using other objective variables as follows: either $\% \mathrm{FEV} 1<80 \%$, chronic cough/phlegm, or respiratory disease treatment; and either $\% \mathrm{FVC}<80 \%$, chronic cough/phlegm, or respiratory disease treatment.

Subtyping of the following comorbidities was performed by hierarchical cluster analysis (Ward's method) as post hoc analysis and stratified by smoking history: overweight, underweight, inflammation, CKD, myocardial infarction, angina pectoris, cerebrovascular disease, osteoporosis, fracture, depression, anxiety disorder, sleep disorder, diabetes mellitus, and lung cancer. Cluster analysis was used to construct an informative classification of an initially unclassified set of data and clusters were constructed so that individuals within clusters were similar and different from individuals in other clusters. ${ }^{24}$ Subject background, lung function (FEV1/FVC, \%FEV1, \%FVC), and CAT score were compared between groups classified by cluster analysis. Differences between the two groups were examined by the chi-square test for categorical data. The measuring data were calculated as the mean and standard deviation (SD), and the Kruskal-Wallis test was performed after checking normality. For variables with significant differences between any groups, a subtest was 
performed to ascertain which clusters differed from which clusters by comparison among multiple clusters. Further analyses were performed to obtain adjusted p-values by the Benjamini \& Hochberg method for categorical data. Multiple comparison analyses were performed by the Steel-Dwass method for measuring data. Statistical analyses were performed using $\mathrm{R}$ version 2.15 .2 ( $\mathrm{R}$ Foundation for Statistical Computing, Vienna, Austria).

\section{Results}

A total of 22,293 subjects were enrolled in this study. Of these, $62.9 \%$ were male, mean (SD) age was 54.6 (9.6) years, $19.3 \%$ were current smokers, $30.2 \%$ were past smokers, and $50.5 \%$ were never-smokers (Table 1 ). There were $962(4.3 \%)$ subjects with airflow limitation. ${ }^{1}$ Three-hundred eighty $(1.7 \%)$ subjects had chronic cough/phlegm (including those who had received current treatment for chronic bronchitis) ${ }^{2}$ and $400(1.8 \%)$ subjects had received current treatment for respiratory diseases listed in the "Study design and participants" section. A total of 1520 (6.8\%) subjects had at least one of the airflow limitation, chronic cough/phlegm, and currently treated respiratory diseases. The breakdown of the 1520 subjects by smoking history and sex was 987 ever-smokers (905 males, 82 females), 525 never-smokers (250 males, 275 females), and 8 subjects with unknown smoking history.

The backgrounds of the subjects according to the presence or absence of airflow limitation, chronic cough/ phlegm, or respiratory diseases are as listed in Table 1. In the multivariate logistic regression analysis, the following explanatory variables were significantly associated with having at least one of airflow limitation, chronic cough/phlegm, and currently treated respiratory diseases: older age, higher total CAT score, and coexistence of lung cancer (common in ever-smokers and never-smokers), higher pack-years, lower BMI, higher CRP, and without coexistence of diabetes mellitus (specific in ever-smokers), male sex, coexistence of anxiety, and sleep disorder (specific in never-smokers) (Table 2). Coexistence of diabetes was negatively associated in both total subjects and eversmokers, but when additional analyses were performed by changing the objective variable from airflow limitation to $\% \mathrm{FEV} 1<80 \%$, it was positively associated in total subjects. However, no association was detected in ever-smokers or never-smokers (Table 3A). By changing the objective variable from airflow limitation to $\% \mathrm{FVC}<$ $80 \%$, coexistence of diabetes was positively associated in the total subjects and never-smokers, and no association was observed in ever-smokers (Table 3B).

A cluster analysis using comorbidities was performed in 1512 subjects with smoking history data who had at least one of the airflow limitation, chronic cough/phlegm, and currently treated respiratory diseases. Each cluster was defined by the presence or absence of comorbidities as follows: In ever-smokers ( $\mathrm{n}=987$ ), cluster SN (smokers/ no comorbidities, $\mathrm{n}=500$ ), SO (smokers/overweight, $\mathrm{n}=130$ ), SC (smokers/CKD, $\mathrm{n}=110$ ), SI (smokers/inflammation, $\mathrm{n}=83$ ) and SM (smokers/mixed, $\mathrm{n}=164)$ were identified. In never-smokers $(\mathrm{n}=525)$, cluster NN (neversmokers/no comorbidities, $\mathrm{n}=249$ ), NC (never-smokers/ CKD, $\mathrm{n}=57$ ), NO (never-smokers/overweight, $\mathrm{n}=56$ ), NU (never-smokers/underweight, $\mathrm{n}=29$ ) and NM (neversmokers/mixed, $n=134$ ) were identified. The results of hierarchical-clustering are presented in dendrograms (Figure 1) by excluding the clusters with no comorbidities in ever-smokers ( $\mathrm{SN})$ and never-smokers $(\mathrm{NN})$.

Figure 2 shows the prevalence of each comorbidity in each cluster in ever-smokers and never-smokers. The cluster SO included those who were overweight only, SC included those with CKD among whom $33.6 \%$ were overweight, SI included those with inflammation among whom $34.9 \%$ were overweight, and SM included those with a mixture of comorbidities. The major comorbidities in the SM cluster were diabetes (30.5\%), CKD (28.0\%), underweight $(23.8 \%)$, overweight $(21.3 \%)$, and inflammation (21.3\%). The cluster NC included those with CKD, NO included those who were overweight, NU included those who were underweight of which $10.3 \%$ had osteoporosis, and NM included those with a mixture of comorbidities. The major comorbidities in the NM cluster were overweight $(37.3 \%)$, inflammation (32.8\%), CKD (29.9\%), diabetes (21.6\%), and sleep disorder (15.7\%).

Demographics and clinical characteristics of each cluster are presented in Table 4A-B. Significant differences between clusters were found in age, BMI, CRP, FEV1/FVC, \%FEV1, $\% \mathrm{FVC}$, and CAT scores among ever-smokers (Table 4A), and in sex, age, BMI, CRP, \%FVC, and CAT scores among never-smokers (Table 4B). Statistical differences between the clusters are shown in Supplementary Table 1 and Supplementary Table 2. Among ever-smokers, the "CKD" cluster was older, the "overweight" cluster had higher BMI and higher FEV1/FVC, and the "inflammation" cluster had higher CRP, compared to the other four clusters (Supplementary Table 1). In never-smokers, the "underweight" cluster had a higher proportion of females, the 
Table I Subject Characteristics by Presence or Absence of Airflow Limitation, Chronic Cough/Phlegm or Currently Treated Respiratory Diseases

\begin{tabular}{|c|c|c|c|c|}
\hline \multirow{2}{*}{\multicolumn{2}{|c|}{ Variable }} & Total & $\begin{array}{l}\text { Having At least One of Airflow } \\
\text { Limitation, Chronic Cough/ } \\
\text { Phlegm, and Currently Treated } \\
\text { Respiratory Diseases }\end{array}$ & $\begin{array}{l}\text { Having Neither Airflow } \\
\text { Limitation, Chronic Cough/ } \\
\text { Phlegm, nor Currently Treated } \\
\text { Respiratory Diseases }\end{array}$ \\
\hline & & $N=22,293$ & $n=1520$ & $n=20,773$ \\
\hline Age (years) & & $54.6 \pm 9.6$ & $60.0 \pm 10.7$ & $54.3 \pm 9.4$ \\
\hline \multirow[t]{2}{*}{ Sex } & Male & $14,0 \mid 3(62.9)$ & II 62 (76.4) & $|2,85|(6 \mid .9)$ \\
\hline & Female & $8280(37.1)$ & $358(23.6)$ & $7922(38.1)$ \\
\hline BMI $\left(\mathrm{kg} / \mathrm{m}^{2}\right)$ & & $23.0 \pm 3.3$ & $23.0 \pm 3.2$ & $23.0 \pm 3.3$ \\
\hline \multirow[t]{4}{*}{ Smoking history } & None & $1 \mathrm{I}, 2 \mathrm{I} 2(50.5)$ & $525(34.7)$ & 10,687 (5I.7) \\
\hline & & $6696(30.2)$ & $570(37.7)$ & $6126(29.6)$ \\
\hline & Current & $4285(19.3)$ & $417(27.6)$ & 3868 (18.7) \\
\hline & Missing data & 100 & 8 & 92 \\
\hline Pack-years & & $11.2 \pm 17.6$ & $20.0 \pm 22.1$ & $10.6 \pm 17.0$ \\
\hline FEVI (L) & & $2.82 \pm 0.68$ & $2.41 \pm 0.68$ & $2.84 \pm 0.67$ \\
\hline \%FEVI (\%) & & $96.0 \pm 14.4$ & $82.6 \pm 17.0$ & $97.0 \pm 13.7$ \\
\hline FEVI/FVC (\%) & & $81.0 \pm 6.3$ & $70.1 \pm 9.4$ & $81.8 \pm 5.2$ \\
\hline FVC (L) & & $3.48 \pm 0.82$ & $3.44 \pm 0.86$ & $3.49 \pm 0.82$ \\
\hline \%FVC (\%) & & $99.2 \pm 13.6$ & $97.4 \pm 16.2$ & $99.3 \pm 13.4$ \\
\hline 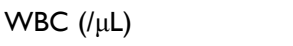 & & $5385 \pm 1,762$ & $5683 \pm 1588$ & $5364 \pm 1772$ \\
\hline CRP (mg/dL) & & $0.11 \pm 0.31$ & $0.15 \pm 0.40$ & $0.11 \pm 0.31$ \\
\hline HbAlc (\%) & & $5.65 \pm 0.62$ & $5.75 \pm 0.67$ & $5.65 \pm 0.61$ \\
\hline eGFR $\left(\mathrm{mL} / \mathrm{min} / 1.73 \mathrm{~m}^{2}\right)$ & & $73.8 \pm 13.0$ & $72.1 \pm 13.6$ & $73.9 \pm 12.9$ \\
\hline CAT score & & $6.5 \pm 4.9$ & $9.0 \pm 6.4$ & $6.3 \pm 4.7$ \\
\hline \multicolumn{5}{|l|}{ Comorbidity } \\
\hline Myocardial infarction & & $80(0.4)$ & $10(0.7)$ & $70(0.3)$ \\
\hline Angina pectoris & & I88 (0.8) & $23(1.5)$ & $165(0.8)$ \\
\hline Cerebrovascular disease & & $|4|(0.6)$ & $13(0.9)$ & $128(0.6)$ \\
\hline Osteoporosis & & $234(1.0)$ & $25(1.6)$ & $209(1.0)$ \\
\hline Fracture & & $48(0.2)$ & $6(0.4)$ & $42(0.2)$ \\
\hline Depression & & $233(1.0)$ & $14(0.9)$ & $219(1.1)$ \\
\hline Anxiety disorder & & $180(0.8)$ & $18(1.2)$ & $162(0.8)$ \\
\hline Sleep disorder & & $428(1.9)$ & $49(3.2)$ & $379(1.8)$ \\
\hline Diabetes mellitus & & $I,|3|(5 . I)$ & $80(5.3)$ & $I, 05 I(5 . I)$ \\
\hline Lung cancer & & $59(0.3)$ & $14(0.9)$ & $45(0.2)$ \\
\hline
\end{tabular}

Notes: Data are shown as numbers (\%) or mean \pm SD. Among the 22,293 subjects, 100 had no smoking data. Each comorbidity was defined as the number of examinees under treatment at the time of examination except for lung cancer. For lung cancer, it was defined as the number of diagnosed examinees, regardless of whether they were under treatment at the time of examination.

Abbreviations: BMI, body mass index; CAT, chronic obstructive pulmonary disease assessment test; CRP, C-reactive protein; eGFR, estimated glomerular filtration rate; FEVI, forced expiratory volume in I s; \%FEVI, percent predicted FEVI; FVC, forced vital capacity; \%FVC, percent predicted FVC; HbAIc, hemoglobin AIc; SD, standard deviation; WBC, white blood cell.

"overweight" cluster had higher BMI, and the "mixed" cluster had higher CRP, compared to the other four clusters (Supplementary Table 2).

\section{Discussion}

In the present study, several factors were identified as associated factors of having at least one of the airflow limitation, chronic cough/phlegm and currently treated respiratory diseases and they differed between ever-smokers and never-smokers. Higher pack-years, lower BMI, and higher CRP, without coexistence of diabetes, were associated factors specific in ever-smokers, whereas male sex, coexistence of anxiety, and sleep disorder were specific in never-smokers. These findings could provide 


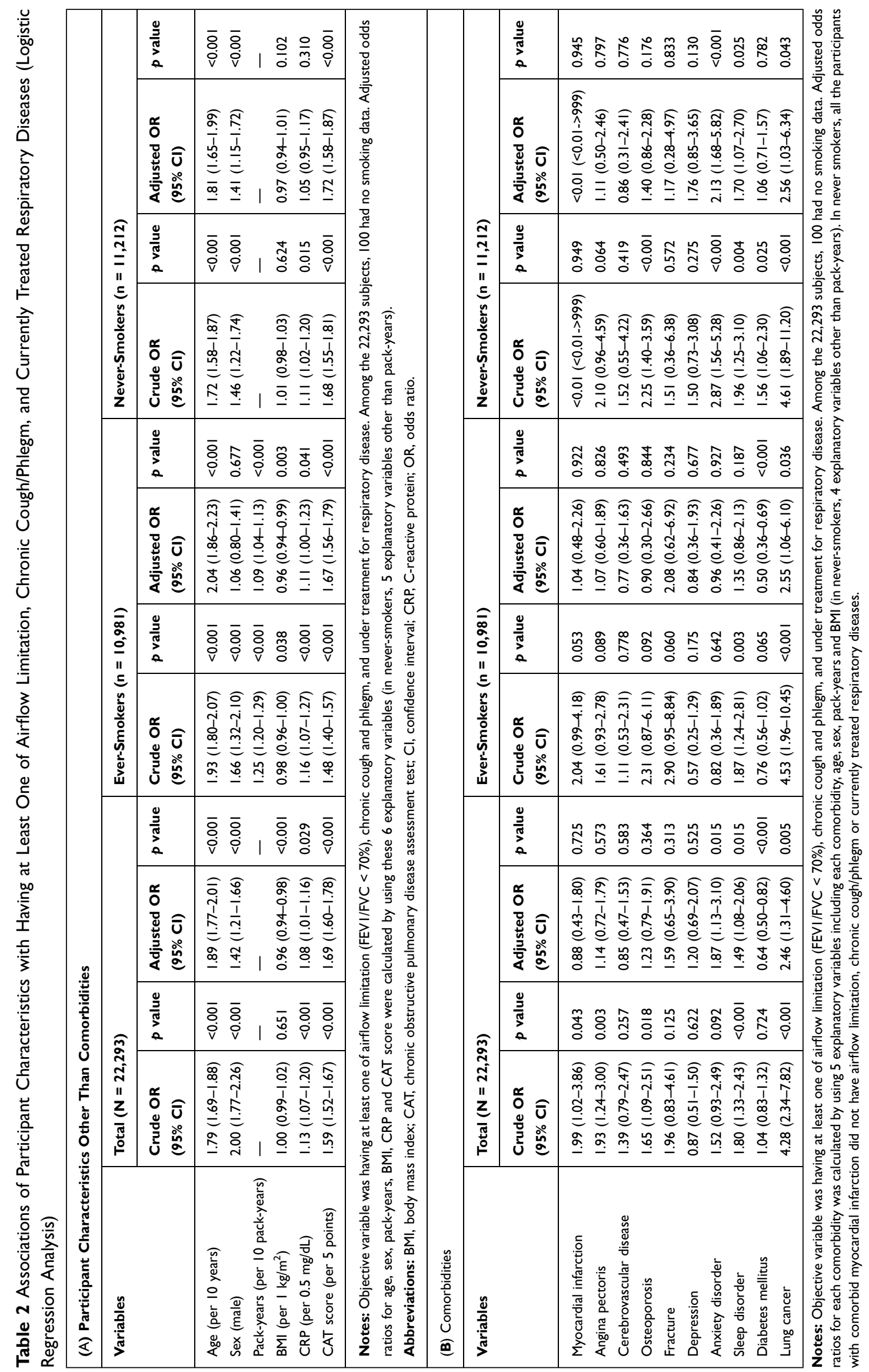


Table 3 Odds Ratios for Comorbid Diabetes Mellitus with Different Objective Variable Settings (Logistic Regression Analyses)

(A) Objective Variable: Having at Least One of \%FEVI < $80 \%$, Chronic Cough/Phlegm, and Currently Treated Respiratory Diseases

\begin{tabular}{|l|l|l|l|l|}
\hline Subjects & Crude OR (95\% Cl) & $\boldsymbol{p}$ value & Adjusted OR (95\% CI) & $\boldsymbol{p}$ value \\
\hline Total & $1.77(\mathrm{I} .52-2.06)$ & $<0.00 \mathrm{I}$ & $1.19(1.02-1.40)$ & 0.028 \\
Ever-smokers & $1.54(1.28-1.86)$ & $<0.00 \mathrm{I}$ & $1.13(0.93-1.37)$ & 0.208 \\
Never-smokers & $1.8 \mathrm{I}(\mathrm{I} .37-2.38)$ & $<0.00 \mathrm{I}$ & $1.32(1.00-1.75)$ & 0.052 \\
\hline
\end{tabular}

(B) Objective Variable: Having at Least One of \%FVC $<80 \%$, Chronic Cough/Phlegm, and Currently Treated Respiratory Diseases

\begin{tabular}{|l|l|l|l|l|}
\hline Subjects & Crude OR (95\% CI) & P value & Adjusted OR (95\% CI) & P value \\
\hline Total & $1.85(1.56-2.19)$ & $<0.001$ & $1.28(1.07-1.53)$ & 0.006 \\
Ever-smokers & $1.59(1.28-1.97)$ & $<0.001$ & $1.18(0.94-1.46)$ & 0.149 \\
Never-smokers & $2.09(1.57-2.77)$ & $<0.001$ & $1.51(1.13-2.02)$ & 0.006 \\
\hline
\end{tabular}

Notes: Explanatory variables for calculating adjusted odds ratios were having comorbid diabetes mellitus, age, sex, pack-years and BMI in ever-smokers, and 4 variables other than pack-years in never-smokers.

Abbreviations: $\mathrm{BMI}$, body mass index; $\mathrm{Cl}$, confidence interval; OR, odds ratio.

information to assist the management of subjects with COPD or at risk for COPD in the general population.

In the total study population, older age, male sex, lower BMI, higher CRP, higher CAT score, coexistence of anxiety, sleep disorder or lung cancer, and without coexistence of diabetes were associated with having at least one of the airflow limitation, chronic cough/phlegm, and currently treated respiratory diseases after adjustment for covariates. Similar associated factors for airflow limitation have been reported in Japanese subjects who went through health examination $^{25}$ and Japanese patients with respiratory diseases. ${ }^{26}$ With regard to the association between age and airflow limitation, a study by the Japanese Respiratory Society demonstrated that FEV1/FVC decreased with age in both sexes. ${ }^{20}$ Lung cancer was prevalent and one of the most common causes of death in COPD patients, ${ }^{27}$ while COPD was an independent risk factor for lung cancer. ${ }^{28} \mathrm{~A}$ study among Japanese in a health examination setting demonstrated that CRP was higher in subjects with airflow limitation with reduced $\%$ FEV1 $(<80 \%)$ compared to those with normal lung function. ${ }^{29}$

In the present study, the coexistence of anxiety or sleep disorder was associated with having at least one of the airflow limitation, chronic cough/phlegm, and currently treated respiratory diseases among never-smokers and in the total population. A systematic review by Atlantis et al showed that anxiety and COPD have a bidirectional association and anxiety increases the risk for COPD in most studies. ${ }^{30}$ Among Japanese COPD patients, CAT total score was higher in patients with anxiety, suggesting a potential association between COPD symptoms and anxiety. ${ }^{31} \mathrm{~A}$ bidirectional association between sleep disorder and COPD has been reported in an article ${ }^{32}$ demonstrating that COPD symptoms disturb sleep and sleep deprivation worsens the clinical presentation of COPD.

Bidirectional association between COPD and diabetes is reported. ${ }^{33}$ Diabetes occurs more often in individuals with COPD than in the general population. Higher blood glucose and insulin resistance are related to impaired lung function. In our study, comorbid diabetes was associated with the absence of airflow limitation, chronic cough/ phlegm or currently treated respiratory diseases in the total population. Airflow limitation refers to reduced FEV1/FVC and thus it does not decrease or even increase if both FEV1 and FVC are reduced. In fact, in the study by Yeh et al, individuals with diabetes had lower FEV1 and FVC but higher FEV1/FVC compared to those without diabetes. ${ }^{34}$ Our study found associations of coexistence of diabetes with reduced $\% \mathrm{FEV} 1$ and reduced $\% \mathrm{FVC}$ in the total population (Table 3), and these results are consistent with the association between comorbid diabetes and the absence of airflow limitation (reduced FEV1/FVC).

In the present study, among subjects having at least one of the airflow limitation, chronic cough/phlegm, and currently treated respiratory diseases, five clusters of comorbidities (no comorbidities, inflammation, overweight, $\mathrm{CKD}$, and mixed) were identified in ever-smokers, and a different set of clusters (no comorbidities, overweight, $\mathrm{CKD}$, underweight, and mixed) were found in never-smokers. In ever-smokers, as compared with the "none" and "CKD" clusters, the 


\section{A}

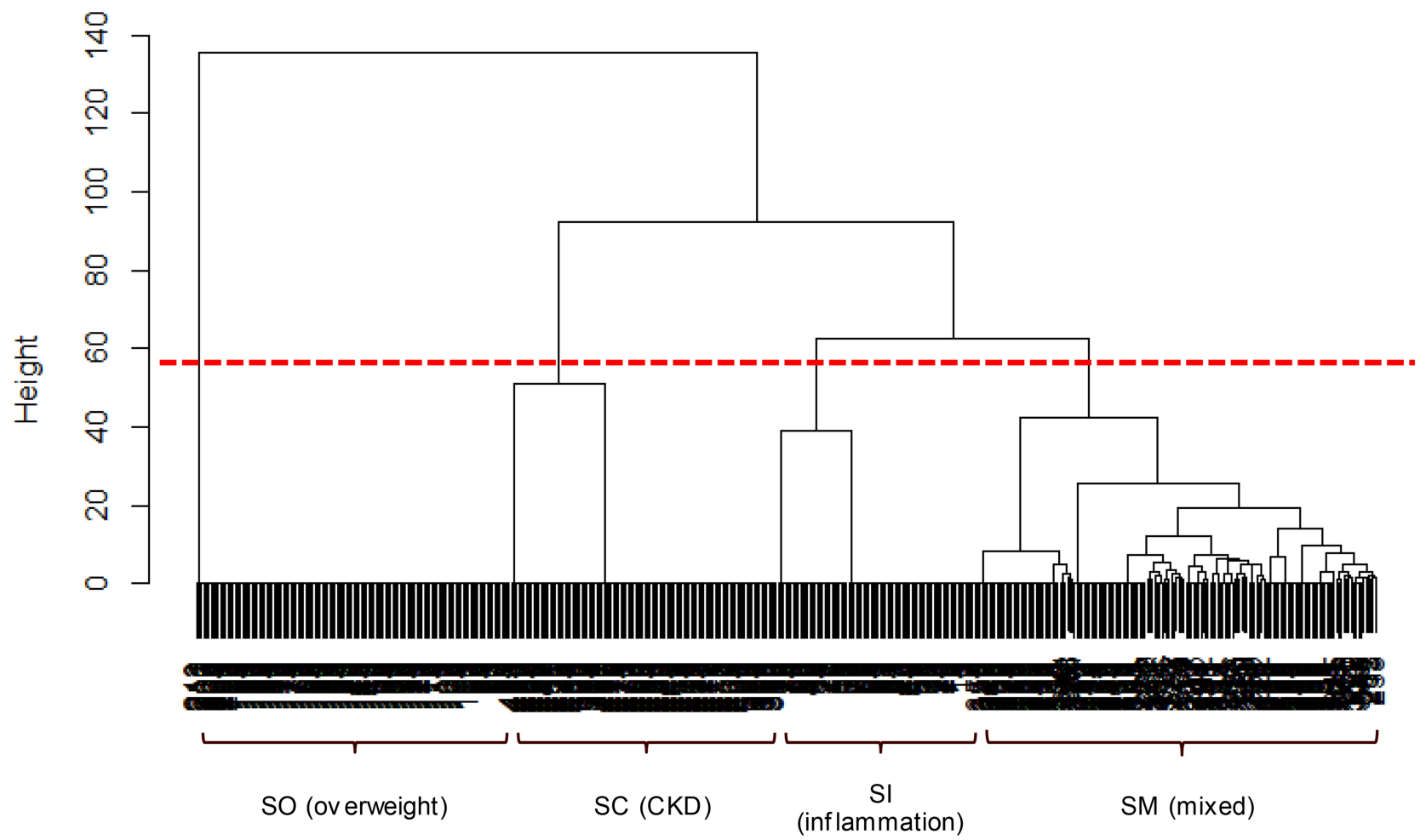

B

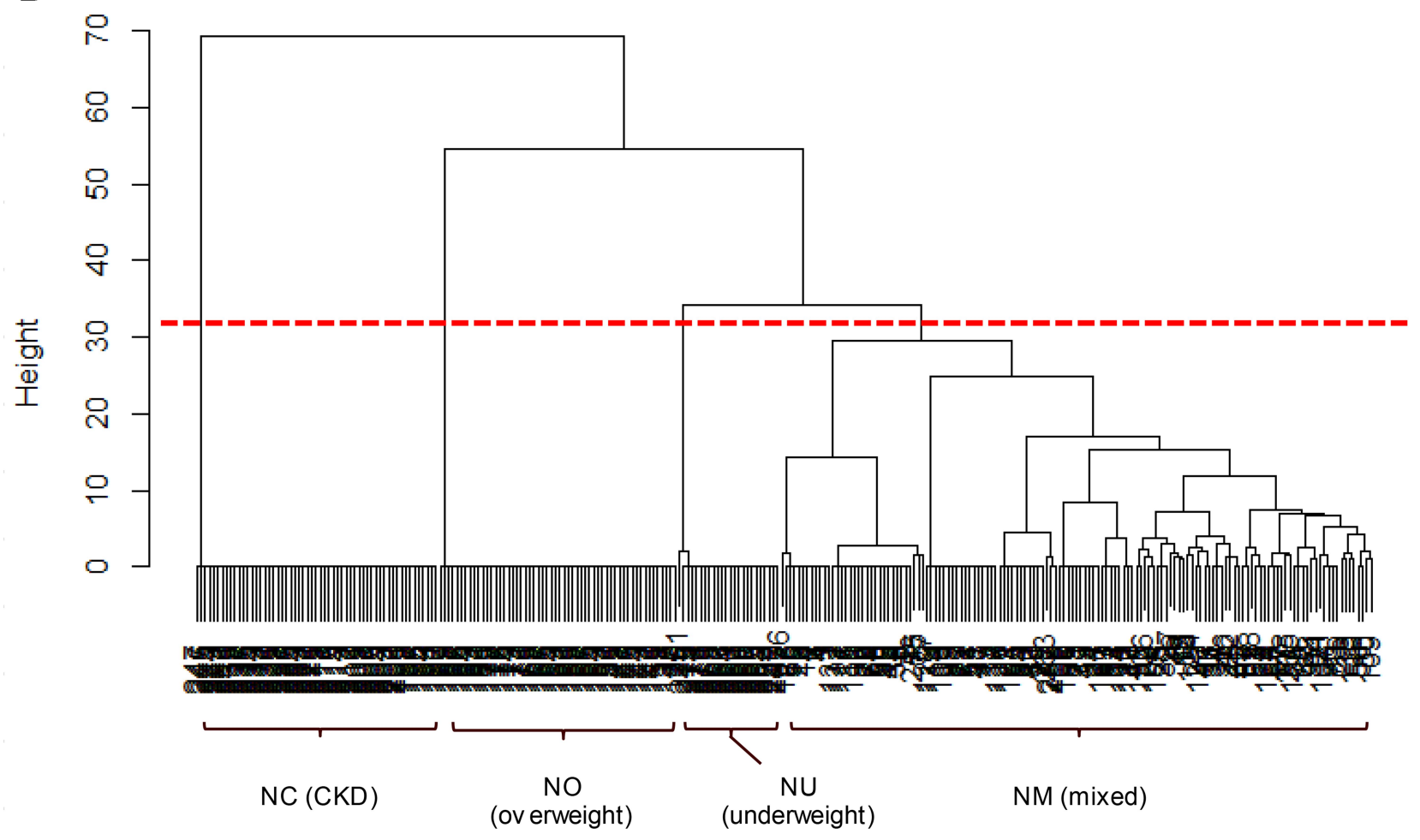

Figure I Cluster dendrogram of subjects by comorbidities. The "height" on the vertical axis indicates the dissimilarity/distance between clusters. The higher "height" represents less similarity between clusters. (A) Ever-smokers $(n=487)$ were first divided into SO cluster (overweight) and others, followed by SC (CKD) and SI (inflammation), in order. $(B)$ Never-smokers $(n=276)$ were first divided into NC $(C K D)$ and others, followed by NO (overweight) and NU (underweight), in order. 

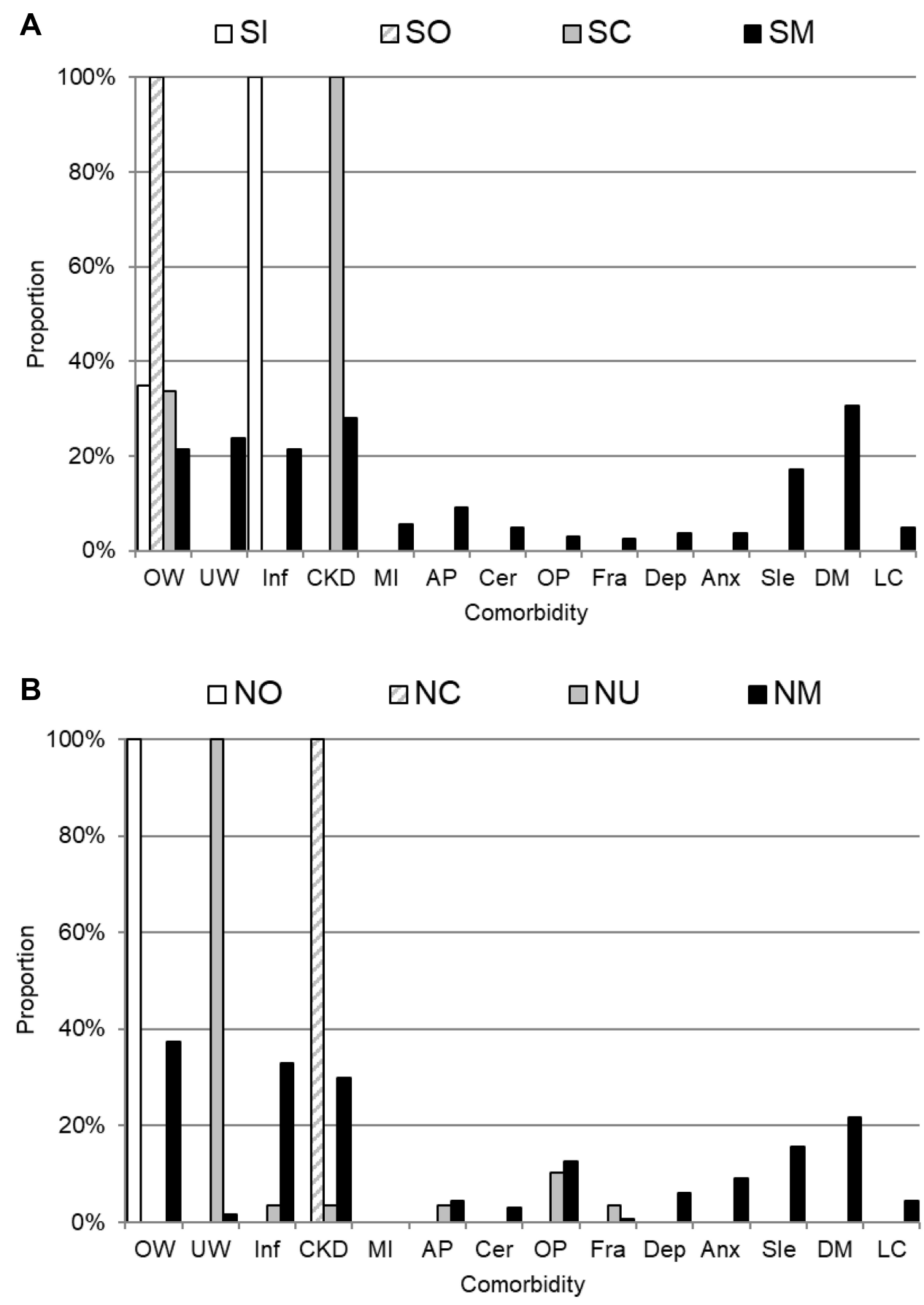

Figure 2 The prevalence of each comorbid disease sorted by the comorbid clusters (excluding no comorbidity cluster) among ever-smokers $(\mathbf{A}$, $\mathrm{n}=487)$ and never-smokers $(B, n=276$ ). For example, among ever-smokers, $34.9 \%$ of SI cluster, $100 \%$ of SO cluster, $33.6 \%$ of SC cluster and $21.3 \%$ of SM cluster had OW (overweight).

Abbreviations: OW, overweight; UW, underweight; Inf, inflammation; CKD, chronic kidney disease; MI, myocardial infarction; AP, angina pectoris; Cer, cerebrovascular disease; OP, osteoporosis; Fra, fracture; Dep, depression; Anx, anxiety disorder; Sle, sleep disorder; DM, diabetes mellitus; LC, lung cancer.

"overweight” cluster had higher FEV1/FVC and higher CAT score. As compared with the "inflammation" and "mixed" clusters, the "overweight" cluster had higher FEV1/FVC, but CAT score was not different. In never-smokers, as compared with the "none" and "CKD" clusters, the "mixed" and "overweight" clusters showed higher CAT scores, whereas \%
FEV1 and FEV1/FVC had no difference. Taken together, lung function characteristics were independent of respiratory symptoms evaluated by the CAT score. Our findings are consistent with the results among COPD patients by Chubachi et $\mathrm{al}^{15}$ in terms that lung function characteristics are independent of respiratory symptoms. In ever-smokers, 
Table 4 Characteristics of Comorbidity-Based Clusters

(A) Characteristics of Each Cluster in Ever-Smokers

\begin{tabular}{|c|c|c|c|c|c|c|}
\hline & $\begin{array}{l}\text { Cluster SN } \\
\text { (None) } \\
n=500\end{array}$ & $\begin{array}{l}\text { Cluster SI } \\
\text { (Inflammation) } \\
\mathbf{n}=\mathbf{8 3}\end{array}$ & $\begin{array}{l}\text { Cluster SO } \\
\text { (Overweight) } \\
n=130\end{array}$ & $\begin{array}{l}\text { Cluster SC } \\
\text { (CKD) } \\
n=110\end{array}$ & $\begin{array}{l}\text { Cluster SM } \\
\text { (Mixed) } \\
n=164\end{array}$ & $p$ value \\
\hline Sex, female & $39(7.8)$ & $6(7.2)$ & $9(6.9)$ & $6(5.5)$ & $22(13.4)$ & 0.116 \\
\hline Age (years) & $58.7 \pm 10.1$ & $57.1 \pm 10.2$ & $56.2 \pm 9.4$ & $66.6 \pm 8.9$ & $63.1 \pm 10.9$ & $<0.001$ \\
\hline BMI $\left(\mathrm{kg} / \mathrm{m}^{2}\right)$ & $22.2 \pm 1.6$ & $24.0 \pm 3.3$ & $27.2 \pm 2.0$ & $24.1 \pm 2.8$ & $22.5 \pm 4.4$ & $<0.001$ \\
\hline CRP (mg/dL) & $0.07 \pm 0.06$ & $0.54 \pm 0.92$ & $0.10 \pm 0.07$ & $0.10 \pm 0.07$ & $0.28 \pm 0.66$ & $<0.001$ \\
\hline FEVI/FVC (\%) & $68.7 \pm 8.9$ & $68.9 \pm 10.0$ & $73.5 \pm 9.1$ & $67.3 \pm 8.8$ & $68.1 \pm 8.3$ & $<0.001$ \\
\hline \%FEVI (\%) & $81.3 \pm 16.0$ & $77.2 \pm 16.7$ & $81.0 \pm 15.2$ & $82.7 \pm 17.9$ & $77.6 \pm 16.9$ & 0.019 \\
\hline \%FVC (\%) & $98.4 \pm 15.2$ & $93.8 \pm 16.4$ & $92.5 \pm 14.3$ & $100.1 \pm 16.9$ & $93.5 \pm 16.3$ & $<0.001$ \\
\hline CAT score & $8.7 \pm 6.0$ & $11.3 \pm 7.4$ & $10.6 \pm 6.4$ & $8.0 \pm 5.6$ & $9.6 \pm 6.8$ & $<0.001$ \\
\hline
\end{tabular}

Note: Data are presented as numbers (\%) or mean \pm SD.

(B) Characteristics of Each Cluster in Never-Smokers

\begin{tabular}{|c|c|c|c|c|c|c|}
\hline & $\begin{array}{l}\text { Cluster NN } \\
\text { (None) } \\
n=249\end{array}$ & $\begin{array}{l}\text { Cluster NO } \\
\text { (Overweight) } \\
n=56\end{array}$ & $\begin{array}{l}\text { Cluster NC } \\
\text { (CKD) } \\
n=57\end{array}$ & $\begin{array}{l}\text { Cluster NU } \\
\text { (Underweight) } \\
\mathbf{n}=\mathbf{2 9}\end{array}$ & $\begin{array}{l}\text { Cluster NM } \\
\text { (Mixed) } \\
n=134\end{array}$ & $p$ value \\
\hline Sex, female & $132(53.0)$ & $29(51.8)$ & $24(42.1)$ & $25(86.2)$ & $65(48.5)$ & 0.002 \\
\hline Age (years) & $57.8 \pm 11.2$ & $57.6 \pm 10.5$ & $66.2 \pm 9.0$ & $58.8 \pm 10.3$ & $63.8 \pm 10.6$ & $<0.001$ \\
\hline BMI $\left(\mathrm{kg} / \mathrm{m}^{2}\right)$ & $21.7 \pm 1.6$ & $27.1 \pm 2.0$ & $22.0 \pm 1.7$ & $17.3 \pm 1.0$ & $24.0 \pm 3.9$ & $<0.001$ \\
\hline CRP (mg/dL) & $0.06 \pm 0.05$ & $0.10 \pm 0.07$ & $0.07 \pm 0.05$ & $0.06 \pm 0.09$ & $0.37 \pm 0.72$ & $<0.001$ \\
\hline FEVI/FVC (\%) & $71.8 \pm 10.0$ & $74.6 \pm 10.2$ & $70.2 \pm 8.7$ & $69.6 \pm 8.1$ & $72.3 \pm 9.8$ & 0.175 \\
\hline \%FEVI (\%) & $88.2 \pm 17.2$ & $85.7 \pm 16.3$ & $86.1 \pm 17.2$ & $84.8 \pm 18.6$ & $84.1 \pm 17.7$ & 0.082 \\
\hline \%FVC (\%) & $101.4 \pm 16.1$ & $95.2 \pm 16.4$ & $99.8 \pm 18.1$ & $99.5 \pm 20.3$ & $94.5 \pm 15.8$ & 0.001 \\
\hline CAT score & $7.5 \pm 5.9$ & $10.5 \pm 6.2$ & $6.6 \pm 5.0$ & $8.3 \pm 6.1$ & $10.5 \pm 7.6$ & $<0.001$ \\
\hline
\end{tabular}

Note: Data are presented as numbers (\%) or mean \pm SD.

Abbreviations: BMI, body mass index; CKD, chronic kidney disease; CRP, C-reactive protein; FEVI, forced expiratory volume in I s; \%FEVI, percent predicted FEVI; FVC, forced vital capacity; \%FVC, percent predicted FVC; CAT, chronic obstructive pulmonary disease assessment test; SD, standard deviation.

higher FEV1/FVC in the "overweight" cluster could be related to lower $\% \mathrm{FVC}$ in the "overweight" cluster, since there were no differences in \%FEV1 between clusters. The "overweight" cluster had higher BMI, and the association between higher BMI and lower FVC was reported among Japanese individuals undergoing health examination. ${ }^{35}$ To our knowledge, the present study is the first report of comorbidity cluster analysis among health examinees having at least one of the airflow limitation, chronic cough/phlegm, and currently treated respiratory diseases and not among diagnosed COPD patients.

The strength of this study is that it was conducted on a large scale by targeting multiple regions in Japan. All the facilities in this study belong to the Japan Society of Ningen Dock and provided standardized and high-quality spirometry testing. There are several limitations to this study. Age and sex distributions of subjects differed from those in the general population because of the setting of the comprehensive health examination, and thus the results need to be interpreted accordingly. Spirometry after bronchodilator inhalation was not performed because the objective of health examination was screening and not a diagnosis. Therefore, subjects found to have airflow limitation included patients with asthma or other respiratory diseases, as well as COPD. Blood pressure and blood lipid data were not included as explanatory variables because we did not investigate the treatment status of hypertension and dyslipidemia and thus blood pressure and blood lipid data were mixed ones with and without treatment. We did not collect data regarding passive smoking and environmental factors (such as occupational factors and air pollution), and we did not evaluate the effects of these factors on having at least one of the airflow limitation, chronic cough/phlegm, and currently treated respiratory diseases. The causal relationship between the explanatory variables and the objective 
variables was unclear because of the cross-sectional design of this study.

\section{Conclusions}

We identified several factors as associated factors of having at least one of the airflow limitation, chronic cough/phlegm, and currently treated respiratory diseases. The associated factors were different between ever-smokers and never-smokers. Different comorbidity patterns were observed by smoking history. Our findings could provide information to assist the management of subjects with COPD or at risk for COPD in the general population.

\section{Abbreviations}

AP, angina pectoris; Anx, anxiety disorder; BMI, body mass index; CAT, chronic obstructive pulmonary disease assessment test; Cer, cerebrovascular disease; CKD, chronic kidney disease; COPD, chronic obstructive pulmonary disease; $\mathrm{CI}$, confidence interval; $\mathrm{Cr}$, serum creatinine; CRP, C-reactive protein; Dep, depression; DM, diabetes mellitus; eGFR, estimated glomerular filtration rate; FEV1/FVC, (forced expiratory volume in $1 \mathrm{~s}$ )/(forced vital capacity); Fra, fracture; GSK, GlaxoSmithKline; HbA1c, hemoglobin A1c; Inf, inflammation; LC, lung cancer; MI, myocardial infarction; NC, never-smokers/CKD; NM, never-smokers/ mixed comorbidities; NN, never-smokers/no comorbidity; NO, never-smokers/overweight; NU, never-smokers/underweight; OP, osteoporosis; OR, odds ratio; OW, overweight; SC, smokers/CKD; SCOPE-J, Survey of Chronic Obstructive pulmonary disease Patients Epidemiology in Japan; SD, standard deviation; SI, smokers/inflammation; Sle, sleep disorder; SM, smokers/mixed comorbidities; SN, smokers/no comorbidity; SO, smokers/overweight; UW, underweight; WBC, white blood cell.

\section{Acknowledgments}

The authors are grateful to the examinees who participated in this survey and the staffs in the medical institutions who contributed to this survey. The following investigators took part in data collection of this survey: Dr. Takeshi Nawa (Hitachi General Hospital, Hitachi Ltd.), Dr. Kazuhiro Gotou (Health Park Clinic Kurosawa), Dr. Takashi Nakagawa (Omiya City Clinic), Dr. Koji Yamashita (Yotsukaido Tokushukai Hospital), Dr. Tatsuo Morikawa (Tokyo-Nishi Tokushukai Hospital), Dr. Takayuki Kashiwabara (JA Shizuoka Kohseiren Enshu Hospital), Dr. Tatsuya Shiraki (Matsubara Tokushukai Hospital), Dr. Ryo
Kobayashi (Bell Clinic), Dr. Hiroshi Sonobe (Chugoku Central Hospital), Dr. Toshiki Fukui (Center for Preventive Medical Treatment, Olive Takamatsu Medical Clinic), Dr. Tokuji Motoki (Kochi Kenshin Clinic), Dr. Yasuhiro Ogata, Dr. Noritaka Higashi (Japanese Red Cross Kumamoto Health Care Center), Dr. Junko Aburaya (Koga Kenshin Center, Koga Ekimae Clinic), and Dr. Koki Ido (Osumi Kanoya Hospital).

\section{Author Contributions}

All authors made a significant contribution to the work reported, whether that is in the conception, study design, execution, acquisition of data, analysis and interpretation, or in all these areas; took part in drafting, revising or critically reviewing the article; gave final approval of the version to be published; have agreed on the journal to which the article has been submitted; and agree to be accountable for all aspects of the work.

\section{Disclosure}

GlaxoSmithKline (GSK) was the funding source and was involved in all stages of this study (WEUSKOP6290). GSK also funded all costs associated with the publishing of the present manuscript. HO, NH, TN, and TF received funding for this study from GSK. TK is an employee of GSK and owns stock of the company; receives pay for part-time lecture from Shiga University of Medical Science. TS is an employee of GSK. The authors report no other potential conflicts of interest for this work.

\section{References}

1. Omori H, Kaise T, Suzuki T, Hagan G. Prevalence of airflow limitation in subjects undergoing comprehensive health examination in Japan: survey of Chronic Obstructive pulmonary disease Patients Epidemiology in Japan. Int $J$ Chron Obstruct Pulmon Dis. 2016;11:873-880.

2. Omori H, Higashi N, Nawa T, Fukui T, Kaise T, Suzuki T. Chronic cough and phlegm in subjects undergoing comprehensive health examination in Japan - survey of Chronic Obstructive pulmonary disease Patients Epidemiology in Japan (SCOPE-J). Int J Chron Obstruct Pulmon Dis. 2020;15:765-773. doi:10.2147/COPD.S237568

3. Salvi SS, Barnes PJ. Chronic obstructive pulmonary disease in non-smokers. Lancet. 2009;374:733-743. doi:10.1016/S0140-6736(09)61303-9

4. Lamprecht B, McBurnie MA, Vollmer WM, et al. COPD in never smokers: results from the population-based burden of obstructive lung disease study. Chest. 2011;139:752-763. doi:10.1378/chest.10-1253

5. Martinez CH, Mannino DM, Divo MJ. Defining COPD-related comorbidities, 2004-2014. Chronic Obstr Pulm Dis. 2014;1:51-63.

6. Divo MJ, Casanova C, Marin JM, et al. COPD comorbidities network. Eur Respir J. 2015;46(3):640-650. doi:10.1183/09031936.00171614

7. Camiciottoli G, Bigazzi F, Magni C, et al. Prevalence of comorbidities according to predominant phenotype and severity of chronic obstructive pulmonary disease. Int $J$ Chron Obstruct Pulmon Dis. 2016;11:2229-2236. doi:10.2147/COPD.S111724 
8. Takahashi S, Betsuyaku T. The chronic obstructive pulmonary disease comorbidity spectrum in Japan differs from that in western countries. Respir Investig. 2015;53:259-270. doi:10.1016/j. resinv.2015.05.005

9. Schwab P, Dhamane AD, Hopson SD, et al. Impact of comorbid conditions in COPD patients on health care resource utilization and costs in a predominantly Medicare population. Int J Chron Obstruct Pulmon Dis. 2017;12:735-744. doi:10.2147/COPD.S112256

10. Park JH, Lee JK, Heo EU, Kim DK, Chung JS. The effect of obesity on patients with mild chronic obstructive pulmonary disease: results from KNHANES 2010 to 2012. Int J Chron Obstruct Pulmon Dis. 2017;12:757-763. doi:10.2147/COPD.S126192

11. Jeong SH, Lee $\mathrm{H}$, Carriere $\mathrm{KC}$, et al. Comorbidity as a contributor to frequent severe acute exacerbation in COPD patients. Int J Chron Obstruct Pulmon Dis. 2016;11:1857-1865. doi:10.2147/COPD. S103063

12. Deniz S, Şengül A, Aydemir Y, Emre JC, Özhan MH. Clinical factors and comorbidities affecting the cost of hospital-treated COPD. Int J Chron Obstruct Pulmon Dis. 2016;11:3023-3030. doi:10.2147/ COPD.S120637

13. Barnes PJ. Inflammatory mechanisms in patients with chronic obstructive pulmonary disease. $J$ Allergy Clin Immunol. 2016;138:16-27. doi:10.1016/j.jaci.2016.05.011

14. Vanfleteren LEGW, Spruit MA, Groenen M, et al. Clusters of comorbidities based on validated objective measurements and systemic inflammation in patients with chronic obstructive pulmonary disease. Am J Respir Crit Care Med. 2013;187:728-735. doi:10.1164/ rccm.201209-1665OC

15. Chubachi S, Sato M, Kameyama N, et al. Identification of five clusters of comorbidities in a longitudinal Japanese chronic obstructive pulmonary disease cohort. Respir Med. 2016;117:272-279. doi:10.1016/j.rmed.2016.07.002

16. Jones PW, Harding G, Berry P, Wiklund I, Chen WH, Leidy NK. Development and first validation of the COPD assessment test. Eur Respir J. 2009;34:648-654. doi:10.1183/09031936.00102509

17. Raghavan N, Lam YM, Webb KA, et al. Components of the COPD assessment test (CAT) associated with a diagnosis of COPD in a random population sample. COPD. 2012;9:175-183. doi:10.3109/ 15412555.2011.650802

18. Nishimura K, Mitsuma S, Kobayashi A, et al. COPD and diseasespecific health status in a working population. Respir Res. 2013;14:61. doi:10.1186/1465-9921-14-61

19. Miller MR, Hankinson J, Brusasco V, et al. Standardization of spirometry. Eur Respir J. 2005;26:319-338. doi:10.1183/ 09031936.05 .00034805

20. Sasaki H, Nakamura M, Kida K, et al. Reference value of spirogram and arterial blood gas partial pressure in Japanese. J Jpn Respir Soc. 2001;39:1-17.

21. Stuart-Harris CH, Crofton J, Gilson JC, et al. Definition and classification of chronic bronchitis for clinical and epidemiological purposes. Lancet. 1965;285:775-779.
22. Japan Society of Ningen Dock. Staging the severity. Available from https://www.ningen-dock.jp/wp/common/data/other/release/hainteiku bun_120405.pdf. Accessed March 31, 2020.

23. Matsuo S, Imai E, Horio M, et al. Revised equations for estimated GFR from serum creatinine in Japan. Am J Kidney Dis. 2009;53:982992. doi:10.1053/j.ajkd.2008.12.034

24. Everitt BS. Commentary: classification and cluster analysis. BMJ. 1995;311(7004):535-536. doi:10.1136/bmj.311.7004.535

25. Masuda S, Omori H, Onoue A, et al. Comorbidities according to airflow limitation severity: data from comprehensive health examination in Japan. Environ Health Prev Med. 2017;22:13. doi:10.1186/ s12199-017-0620-0

26. Nishida Y, Takahashi Y, Tezuka K, et al. A comprehensive analysis of association of medical history with airflow limitation: a cross-sectional study. Int J Chron Obstruct Pulmon Dis. 2017;12:2363-2371. doi:10.2147/COPD.S138103

27. Vestbo J, Hurd SS, Agusti AG, et al. Global strategy for the diagnosis, management, and prevention of chronic obstructive pulmonary disease: GOLD executive summary. Am J Respir Crit Care Med. 2013;187:347-365. doi:10.1164/rccm.201204-0596PP

28. De Torres JP, Marin JM, Casanova C, et al. Lung cancer in patients with chronic obstructive pulmonary disease: incidence and predicting factors. Am J Respir Crit Care Med. 2011;184:913-919. doi:10.1164/ rccm.201103-0430OC

29. Funakoshi Y, Omori H, Mihara S, Marubayashi T, Katoh T. Association between airflow obstruction and the metabolic syndrome or its components in Japanese men. Inter Med. 2010;49:2093-2099. doi:10.2169/internalmedicine.49.3882

30. Atlantis E, Fahey P, Cochrane B, Smith S. Bidirectional associations between clinically relevant depression or anxiety and COPD. Chest. 2013;144(3):766-777. doi:10.1378/chest.12-1911

31. Miyazaki M, Nakamura H, Chubachi S, et al. Analysis of comorbid factors that increase the COPD assessment test scores. Respir Res. 2014;15:13. doi:10.1186/1465-9921-15-13

32. Omachi TA, Blanc PD, Claman DM, et al. Disturbed sleep among COPD patients is longitudinally associated with mortality and adverse COPD outcomes. Sleep Med. 2012;13:476-483. doi:10.1016/j.sleep.2011.12.007

33. Rogliani P, Luca G, Lauro D. Chronic obstructive pulmonary disease and diabetes. COPD Res Pract. 2015;1:3. doi:10.1186/s40749-0150005-y

34. Yeh HC, Punjabi NM, Wang NY, et al. Cross-sectional and prospective study of lung function in adults with type 2 diabetes. Diabetes Care. 2008;31:741-746. doi:10.2337/dc07-1464

35. Goto Y, Yokokawa H, Fukuda H, Naito T, Hisaoka T, Isonuma H. Body mass index and waist circumference are independent risk factors for low vital capacity among Japanese participants of a health checkup: a single-institution cross-sectional study. Environ Health Prev Med. 2015;20:108-115.

\section{Publish your work in this journal}

The International Journal of COPD is an international, peer-reviewed journal of therapeutics and pharmacology focusing on concise rapid reporting of clinical studies and reviews in COPD. Special focus is given to the pathophysiological processes underlying the disease, intervention programs, patient focused education, and self management protocols. This journal is indexed on PubMed Central, MedLine and CAS. The manuscript management system is completely online and includes a very quick and fair peer-review system, which is all easy to use. Visit http://www.dovepress.com/testimonials.php to read real quotes from published authors. 